Cita: Camargo, E.M; López-Gil, J.F; Campos, W., 2021. Comparação das barreiras percebidas para a prática de atividade física de acordo com o sexo e nível de atividade física. Cuadernos de Psicología del Deporte, 21(1), 204-215

\title{
Comparação das barreiras percebidas para a prática de atividade física de acordo com o sexo e nível de atividade física
}

\section{Comparison of perceived barriers to physical activity according to sex and physical activity level}

\section{Comparación de las barreras percibidas para la práctica de actividad física de acuerdo con el sexo y el nivel de actividad física}

\author{
Camargo, E.M. ${ }^{1}$; López-Gil, J.F. ${ }^{2}$; Campos, W. ${ }^{1},{ }^{1}$ Universidade Federal do Paraná (UFPR), Curitiba, \\ Brasil. ${ }^{2}$ Universidad de Murcia (UM), Murcia, España.
}

\section{RESUMO}

Objetivo: Relatar as barreiras percebidas para a prática de atividade física no lazer pelos adolescentes e comparar de acordo com o sexo e nível de atividade física. Métodos: Estudo transversal, conduzido em 2018 com uma amostra representativa de 1518 adolescentes. As barreiras e o nível de atividade física foram avaliados por meio de instrumentos validados. Os adolescentes foram separados em três grupos: inativos, insuficientemente ativos, ativos. Resultados: Meninas reportaram com mais frequência: "não ter companhia" $(33.2 \% ; 34.9 \% ; 31.9 \%)$ e "preguiça" $(30.0 \% ; 38.7 \% ; 31.3 \%)$, sendo respectivamente: inativas, insuficientemente ativas e ativas. "Acha chato", "clima" e "não ter como pagar" apresentaram diferenças estatísticas entre os grupos. Meninos inativos fisicamente reportaram com mais frequência: "não ter alguém para levar" (30.8\%), "preguiça" e "não ter companhia" (30.1\%, ambas). Insuficientemente ativos "não ter companhia" (37.6\%) e "clima" (38.6\%). Ativos reportaram "falta de locais" (38.7\%) e "não ter alguém para levar" (36.2\%). Apenas a barreira "não ter motivação" apresentou diferença significativa. Conclusão: "Não ter companhia" e "preguiça" foram as barreiras mais citadas por meninas em todos os grupos. Meninas insuficientemente ativas apresentaram diferenças significativas, para: "acha chato", "clima" e "não tem como pagar". Meninos reportaram com mais frequência as barreiras: "não ter alguém para levar", "preguiça", "não ter companhia", "clima", "falta de locais". Quando comparado com os demais grupos apenas a barreira "não ter motivação" apresentou diferença significativa para o grupo de meninos.

Palavras chave: barreiras, atividade física, adolescentes.

\section{ABSTRACT}

Objective: To report the barriers perceived by adolescents to engage in physical activity in their free time and compare them according to gender and physical activity level. Methods: A cross-sectional study was conducted in 2018 with a representative sample of 1518 adolescents. Barriers and level of physical activity were assessed using validated instruments. Adolescents were separated into three groups: inactive, insufficiently active and active. Results: Girls reported most frequently: "not having company" $(33.2 \% ; 34.9 \% ; 31.9 \%)$ and "laziness" $(30.0 \%$; $38.7 \% ; 31.3 \%$ ), for inactive, insufficiently active and active groups, respectively. "Finds it boring", "climate" and 


\section{Barreiras para prática de atividade física}

"can't afford it" presented statistical differences between the groups. Physically inactive children most often reported: "not having someone to drive them" (30.8\%), "laziness" and "not having company" (30.1\%, both); for the insufficiently active "not having company" (37.6\%) and "weather" (38.6\%). The active ones reported "lack of places" (38.7\%) and "no one to carry him/her" (36.2\%). Only the "lack of motivation" barrier showed a significant difference. Conclusion: "Not having company" and "laziness" were the barriers most often cited by girls in all groups. Insufficiently active girls showed significant differences for: "finds it boring", "weather" and "can't afford it". Boys reported most often the barriers: "no one to carry him", "laziness", "not having company", "weather", and "lack of places". When the different physical activity groups were compared, only the barrier "lack of motivation" showed a significant difference in boys.

Keywords: barriers, physical activity, adolescents.

\section{RESUMEN}

Objetivo: Reportar las barreras percibidas por los adolescentes para realizar actividad física en el tiempo libre y compararlas según el sexo y el nivel de actividad física. Métodos: Estudio transversal, realizado en 2018 con una muestra representativa de 1518 adolescentes. Las barreras y el nivel de actividad física se evaluaron utilizando instrumentos validados. Los adolescentes fueron separados en tres grupos: inactivos, insuficientemente activos y activos. Resultados: Las niñas reportaron con mayor frecuencia: "no tener compañía" $(33.2 \% ; 34.9 \% ; 31.9 \%)$ y "pereza" $(30.0 \% ; 38.7 \% ; 31.3 \%)$, para los grupos inactivas, insuficientemente activas y activas, respectivamente. "Lo encuentra aburrido", "clima" y "no tener como pagarlo" presentaron diferencias estadísticas entre los grupos. Los niños físicamente inactivos reportaron con mayor frecuencia: "no tener quien lo lleve" (30.8\%), "pereza" y "no tener compañía" (30.1\%, ambos); para los insuficientemente activos "no tener compañía" (37,6\%) y "clima" (38,6\%). Los activos reportaron "falta de lugares" (38.7\%) y "no tener quien lo lleve" (36.2\%). Sólo la barrera de "falta de motivación" mostró diferencia significativa. Conclusión: "No tener compañía" y "pereza" fueron las barreras más citadas por las niñas de todos los grupos. Las niñas insuficientemente activas mostraron diferencias significativas para: "encontrarlo aburrido", "clima" y "no tener como pagarlo". Los chicos reportaron con mayor frecuencia las barreras: "no tener quien lo lleve", "pereza", "no tener compañía", "clima", y "falta de lugares". Cuando los distintos grupos de actividad física fueron comparados, sólo la barrera "falta de motivación" mostró una diferencia significativa en los niños.

Palabras clave: barreras, actividad física, adolescentes.

\section{INTRODUÇÃO}

$\mathrm{O}$ número de crianças e adolescentes obesos em todo o mundo aumentou dez vezes nas últimas quatro décadas e, se as tendências atuais continuarem, haverá, até 2022, mais crianças e adolescentes com obesidade do que com desnutrição moderada e grave (NCD Risk Factor Collaboration, 2017). Segundo esse estudo, de prevalência mundial, que analisou cerca de 130 milhões de pessoas com mais de cinco anos de idade (31,5 milhões de pessoas entre os cinco e os 19 anos e 97,4 milhões com mais de 20 anos). As taxas de obesidade em crianças e adolescentes em todo o mundo aumentaram de menos de $1 \%$ (equivalente a cinco milhões de meninas e seis milhões de meninos) em 1975 para quase 6\% em meninas (50 milhões) e quase $8 \%$ em meninos (74

milhões) em 2016. Combinado, o número de obesos com idade entre cinco e 19 anos cresceu mais de dez vezes, de 11 milhões em 1975 para 124 milhões em 2016. Outros 213 milhões estavam com sobrepeso em 2016, mas o número caiu abaixo do limiar para a obesidade (NCD Risk Factor Collaboration, 2017).

A Organização Mundial da Saúde (OMS) incentiva os países a implementar esforços para abordar os ambientes que hoje estão aumentando a chance de obesidade em nossas crianças. Os países devem procurar particularmente reduzir o consumo de alimentos baratos, ultra processados, densos em calorias e pobres em nutrientes. Também devem reduzir o tempo que as crianças passam em atividades de lazer sedentárias, promovendo uma maior participação em atividades físicas (Organização Mundial da Saúde, [OMS], 2018). 


\section{Camargo; López-Gil \& Campos}

Sobre os níveis de atividade física, o maior estudo mundial que envolveu crianças e adolescentes, publicado por Guthold e colaboradores (2020), analisou a evolução entre 2001 e 2016 de 1,6 milhão de jovens estudantes em quase 300 pesquisas de âmbito escolar em 146 países e territórios. Oito em cada dez crianças e adolescentes de 11 a 17 anos não realizam atividade física suficiente. No Brasil, $84 \%$ dos adolescentes são menos ativos do que deveriam. Os dados mostram ainda que não houve nenhuma melhora significativa, no mundo, nesses níveis nos últimos 15 anos (Guthold et al., 2020).

Segundo Guthold e colaboradores (2020), enquanto $78 \%$ dos meninos brasileiros fazem menos exercício do que deveriam, o percentual é de $89 \%$ entre as meninas, uma diferença de 11 pontos percentuais. Apenas um em cada três países pesquisados registraram diferença de mais de 10 pontos percentuais entre os sexos (Guthold et al., 2020).

Os fatores que podem influenciar a prevalência de inatividade física em adolescentes são muito variados, englobando indicadores sociodemográficos, econômicos ou de apoio social / familiar, entre outros (Portela-Pino et al., 2020; Prochnow et al., 2019; Vasquez et al., 2020). Nesse sentido, investigar as barreiras que impedem a prática de atividade física nessa população parece ser fundamental. Uma vez que um fator chave para o sucesso de programas e intervenções em promoção de atividade física, seja em níveis individuais ou comunitários, está em identificar os aspectos que limitam a participação e manutenção dos indivíduos nesses programas e intervenções (Rech et al., 2018). Assim, identificar se a percepção de barreiras difere em relação a sexo e nível de atividade física pode contribuir para que ações mais específicas possam ser elaboradas.

Dessa maneira, o estudo teve como objetivo relatar as barreiras percebidas para a prática de atividade física no lazer pelos adolescentes e comparar de acordo com o sexo e nível de atividade física (inativo, insuficientemente ativo, ativo).

\section{MATERIAL E MÉTODOS}

Trata-se de um estudo com delineamento transversal realizado no ano de 2018 na cidade de Curitiba, Paraná, Brasil. Para realizar a comparação das barreiras percebidas de acordo com os grupos: sexo e nível de atividade física (inativo, insuficientemente ativo, ativo) foi considerado o desenho de grupos naturais (DGN) (Ato et al., 2013) da área da psicologia aplicada. Cujo objetivo é comparar uma ou mais variáveis dependentes, de grupos preexistentes, onde os grupos são selecionados usando variáveis individuais (ex: sexo, grupo étnico, transtorno psicológico, inteligência, hábitos diários) e pertencem a uma mesma cultura (Ato et al.,2013). Quando os grupos pertencem a culturas diferentes o resultado é o desenho transcultural (DTC) (Ato et al., 2013).

\section{Participantes}

Amostra representativa de adolescentes, com idade de 15 a 18 anos, matriculados no ensino médio da rede estadual de Curitiba/PR. Para garantir a representatividade as escolas estaduais foram estratificadas de acordo com cada uma das nove regiões administrativas do município. Após foi realizado um sorteio de duas escolas em cada uma das nove regiões administrativas da cidade. Seguido de uma seleção aleatória simples de uma turma de cada ano, de acordo com a quantidade de adolescentes, separados por sexo, necessária para determinada região. Por fim, todos os alunos de cada turma foram convidados a participar do estudo.

Foram excluídos aqueles que apresentaram limitações físicas e/ou cognitivas que limitassem a prática de atividade física (informado pelo adolescente). Os adolescentes que não apresentaram o TCLE assinado pelos pais ou responsáveis, aqueles que se negaram a participar do estudo, ou faltaram no dia da coleta, assim como os que responderam os questionários incorretamente, foram considerados como perda amostral. Assim, a amostra analítica do estudo foi de 1518 adolescentes (feminino=59.2\% $\mathrm{n}=899 ;$ masculino $=40,8 \%-\mathrm{n}=619$ ). 


\section{Barreiras para prática de atividade física}

\section{Instrumentos}

O nível de atividade física foi mensurado através do questionário IPAQ - versão curta (Questionário Internacional de Atividade Física), no qual os adolescentes reportavam "quantos dias por semana" e "quanto tempo por dia" praticavam AFMV (atividade física moderada vigorosa) no lazer, ao longo de uma semana habitual (Guedes et al., 2005). O instrumento apresentou reprodutibilidade boa para adolescentes $\geq 14$ anos para atividades moderadas vigorosas (Guedes et al., 2005). Para análise, os adolescentes foram agrupados de acordo com o nível de AFMV (minutos/semana) que reportaram no questionário, sendo: grupo inativos $\leq 10$ minutos/semana, grupo insuficientemente ativos de $>10$ minutos/semana até 419 minutos na semana, grupo ativos fisicamente $\geq 420$ minutos na semana (Tremblay et al., 2016).

A avaliação das barreiras percebidas à prática de atividade física no lazer foi realizada por meio de um instrumento desenvolvido e validado para adolescentes da cidade de Curitiba, Paraná (reprodutibilidade variou entre $\mathrm{ICC}=0,58$ e 0,87 $\alpha=0,75$ e 0,53 ) (Santos et al., 2009). O instrumento avalia 12 barreiras, a saber: "falta de locais", "não conheço lugares", "amigos moram longe”, "não tem alguém para levar", "clima”, "preferência por outras atividades", "preguiça", "falta de motivação", "muitas tarefas", "falta de tempo", "falta de companhia" e "em casa ninguém faz". Cada item do instrumento possui quatro opções de resposta (likert): discordo muito, discordo, concordo e concordo muito (Santos et al., 2009). Para análise das frequências (sexo e grupos) os adolescentes foram classificados de acordo com a ausência (discordo muito ou discordo) ou presença (concordo ou concordo muito) de cada barreira (Santos et al., 2010).

O sexo foi auto reportado ("masculino", "feminino"), a idade decimal calculada a partir da data de nascimento (informada pelo adolescente) subtraída da data de coleta dos dados e dividida por 365, posteriormente a idade foi classificada em " 15 anos", "16 anos", "17 anos", “18 anos". O estado nutricional calculado com os dados mensurados de massa corporal e a estatura $\left(\mathrm{kg} / \mathrm{estatura}^{2}\right)$ e os adolescentes classificados, pelo escore Z da amostra, em "peso normal" e "excesso de peso" (OMS, 2006). A escolaridade dos país e responsáveis, e o nível socioeconômico (NSE) foram avaliados com um questionário padronizado (ABEP, 2016). A escolaridade dos pais foi classificada em: Até Fundamental Completo, Ensino Médio Completo, Ensino Superior Completo (foi perguntado a escolaridade do pai, da mãe e, indicado o chefe da família para cálculo do NSE). O NSE foi classificado em três categorias: "baixo" (classe $\mathrm{C}+\mathrm{D}$ ), "médio" (classe B1 +B2) e "alto" (classe A1+A2) (ABEP, 2016).

\section{Procedimentos}

$\mathrm{O}$ estudo seguiu as normas de pesquisa envolvendo seres humanos do Conselho Nacional de Saúde (resolução n 466/2012), sendo aprovado pelo Comitê de Ética em Pesquisa da Universidade Federal do Paraná (CAAE: 98133218.8.0000.0102) e autorizado pelos pais ou responsáveis dos adolescentes através do termo de consentimento livre e esclarecido. A coleta foi realizada em sala de aula, onde os adolescentes responderam os questionários com a ajuda de pesquisadores treinados.

\section{Análise estatística}

Foi utilizada a estatística descritiva para distribuição das frequências em relação ao sexo e grupos de atividade física (inativo, insuficientemente ativo, ativo). O qui-quadrado foi utilizado para verificar diferenças nas proporções em relação ao sexo e o Kruskal-Wallis para verificar diferenças entre os grupos de AF. Após, através do comando de comparações múltiplas (pós-hoc de Kruskal-Wallis) foi verificado qual grupo apresentou diferença significativa. Foi adotado um nível de significância de $5 \%$ e utilizado o programa SPSS, versão 21.0 para análise dos dados. 


\section{Camargo; López-Gil \& Campos}

\section{RESULTADOS}

Participaram do estudo um total de 1518 adolescentes (feminino $=59.2 \% \quad-\quad n=899$; masculino $=40,8 \%-\mathrm{n}=619)$, com idades entre 15 e 18 anos, sendo: do sexo feminino $61.4 \%(n=285)$ e do sexo masculino 38,6\% $(n=179)$ da amostra de 15 anos; $69.9 \%(n=350)$ sexo feminino e $30,1 \%(n=151)$ do sexo masculino da amostra de 16 anos; $57.4 \%(n=244)$ sexo feminino e $42,6 \%(n=181)$ do sexo masculino da amostra de 17 anos; e $15.6 \%(\mathrm{n}=20)$ sexo feminino e $84,4 \%(n=113)$ do sexo masculino da amostra de 18 anos.

Em relação ao estado nutricional $83 \%$ $(\mathrm{n}=1260)$ da amostra apresentou peso normal, sendo: $61.9 \%(\mathrm{n}=780)$ dessa amostra feminino e $38,1 \%$ $(\mathrm{n}=480)$ do sexo masculino. Em relação ao nível socioeconômico $62.4 \%$ da amostra, a maior parte, relatou pertencer ao nível socioeconômico médio, sendo: $60 \%(n=568)$ dessa amostra do sexo feminino e $40 \%(n=379)$ do sexo masculino. A escolaridade dos pais apresentou a maior parte da amostra de pais (41.4\%, n=628) e mães (41.5\%, $\mathrm{n}=630)$ com escolaridade "até o ensino médio completo".

As barreiras mais frequentes para ambos os grupos foram: "preguiça" ( $\mathrm{n}=633-41.7 \%)$, "não tem companhia" ( $\mathrm{n}=614$ - 40.4\%) e "clima" ( $\mathrm{n}=613)$ $40.4 \%$ ); Por outro lado, os menos frequentes foram "não pode realizar os movimentos" ( $\mathrm{n}=170-11.2 \%) \mathrm{e}$ "não tem como pagar" ( $\mathrm{n}=283$ - 18.6\%). Em relação ao gênero, as meninas relataram como principais barreiras: "preguiça" ( $\mathrm{n}=470-52.3 \%)$, "clima" ( $\mathrm{n}=437$ - 48.6\%) e "não tem companhia" ( $n=428-69.7 \%)$. Para meninos: "não tem locais" ( $\mathrm{n}=186-30.0 \%)$, "não tem ninguém para levar" ( $\mathrm{n}=185$ - 29.9\%) e "falta de instalações " $(\mathrm{n}=181-29.2 \%)$ foram as mais percebidas (Tabela 1). A Tabela 2 apresenta as características da amostra (sexo, idade, estado nutricional, escolaridade pai e mãe, NSE) de acordo com os grupos de atividade física.

Em relação aos grupos de atividade física (Tabela 3), meninas inativas fisicamente reportaram com mais frequência as barreiras: "não ter companhia" $(n=204-33.2 \%)$ e "preguiça" $(n=141-30.0 \%)$.
Assim como, as meninas dos grupos insuficientemente ativas e ativas fisicamente, sendo: "não ter companhia" ( $\mathrm{n}=214-34.9 \%$ vs $\mathrm{n}=196-31.9 \%) \mathrm{e}$ "preguiça" ( $\mathrm{n}=182$ - 38.7\% vs $\mathrm{n}=147-31.3 \%)$, respectivamente.

Meninos inativos fisicamente reportaram com mais frequências as barreiras: "não ter alguém para levar" $(\mathrm{n}=57$ - 30.8\%) e "preguiça e não ter companhia" $\quad(\mathrm{n}=56-30.1 \%$, ambas $)$. Insuficientemente ativos reportaram com mais frequência "não ter companhia" ( $\mathrm{n}=70-37.6 \%)$, seguido de "clima" ( $\mathrm{n}=68-38.6 \%)$. Por fim, o grupo de meninos ativos fisicamente reportaram "falta de locais" ( $\mathrm{n}=70$ - 38.7\%) e "não ter alguém para levar" $(n=67-36.2 \%)$ com mais frequência quando comparado as demais barreiras.

Quando comparado diferenças estatísticas entre os grupos de meninas, as barreiras: "acha chato" ( $p=.013$ insuficientemente ativas), "clima" ( $p=.003$ insuficientemente ativas) e "não ter como pagar" ( $p=.005$ insuficientemente ativas) apresentaram diferenças estatísticas em relação aos grupos. Para meninos, apenas a barreira "não ter motivação" ( $p=.005$ inativos fisicamente) apresentou diferença significativa. 
Tabela 1. Barreiras percebidas para a prática de atividade física no lazer segundo o sexo (Curitiba, Paraná, Brasil $-\mathrm{n}=1518$ ).

\begin{tabular}{l|ccccccc}
\hline \multirow{2}{*}{ Barreiras } & \multicolumn{2}{|c}{ Masculino } & \multicolumn{2}{c}{ Feminino } & \multicolumn{3}{c}{ Total } \\
& $\mathrm{n}$ & $\%$ & $\mathrm{n}$ & $\%$ & $p$ & $\mathrm{n}$ & $\%$ \\
\hline 1 Falta de locais & 181 & $43.0 \%$ & 318 & $57.0 \%$ & $.012 \neq$ & 499 & $100.0 \%$ \\
2 Não tem alguém para levar & 185 & $41.8 \%$ & 295 & $58,2 \%$ & .228 & 480 & $100.0 \%$ \\
3 Não consegue realizar os & 51 & $30.0 \%$ & 119 & $70.0 \%$ & $.002^{*}$ & 170 & $100.0 \%$ \\
movimentos & 160 & $27.7 \%$ & 418 & $72.3 \%$ & $<.001^{*}$ & 578 & $100.0 \%$ \\
4 Prefere fazer outras coisas & 111 & $28.8 \%$ & 275 & $71.2 \%$ & $<.001^{*}$ & 386 & $100.0 \%$ \\
5 Não tem motivação & 163 & $25.8 \%$ & 470 & $74.2 \%$ & $<.001^{*}$ & 633 & $100.0 \%$ \\
6 Tem preguiça & 186 & $30.3 \%$ & 428 & $69.7 \%$ & $<.001^{*}$ & 614 & $100.0 \%$ \\
7 Não tem companhia & 91 & $28.0 \%$ & 234 & $72.0 \%$ & $<.001^{*}$ & 325 & $100.0 \%$ \\
8 Acha chato & 140 & $35.4 \%$ & 256 & $64.6 \%$ & $.011 \neq$ & 396 & $100.0 \%$ \\
9 Ninguém convida & 145 & $33.0 \%$ & 294 & $67.0 \%$ & $<.001^{*}$ & 439 & $100.0 \%$ \\
10 Não tem tempo & 176 & $28.7 \%$ & 437 & $71.3 \%$ & $<.001^{*}$ & 613 & $100.0 \%$ \\
11 O clima dificulta & 78 & $27.6 \%$ & 205 & $72.4 \%$ & $<.001^{*}$ & 283 & $100.0 \%$ \\
12 Não tem como pagar & &
\end{tabular}

Estatisticamente significativo para o teste de qui-quadrado, sendo: $\neq p<.050$ e $* p<.010$.

\section{DISCUSSÃO}

Investigar barreiras para atividade física em adolescentes pode ser primordial para desenvolver abordagens adequadas para reduzir a inatividade física (Portela-Pino et al., 2020; Prochnow et al., 2019; Rech et al., 2018; Vasquez et al., 2020). Apresentar as barreiras percebidas pelos adolescentes de acordo com o nível de atividade física pode ampliar o conhecimento sobre o tema.

Em relação ao sexo, meninas reportaram com mais frequência as barreiras: não ter companhia e preguiça, para todos os grupos (inativo, insuficientemente ativo, ativo). Em relação a preguiça, os resultados concordam com outros estudos da literatura que investigaram amostra de adolescentes (Dias et al., 2015; Portela-Pino et al., 2020 ) embora se distanciem de outros estudos que apontaram para a falta de interesse ou conhecimento para realizar exercícios é mais presente em meninas (Rosselli et al., 2020; Vasquez et al., 2020).

A falta de companhia é uma barreira citada por adolescentes (Prochnow et al., 2019; Rech et al., 2018). Na adolescência é comum iniciar uma independência da família, as relações pessoais com amigos são fortalecidas e suas opiniões são relevantes na tomada de decisões relacionadas ao comportamento, nesse específico, sobre o comportamento ativo ou não (Piola et al., 2019; Prochnow et al., 2019). Sendo assim, ter companhia dos amigos para realizar atividade física no lazer parece ser importante na decisão de realizar a prática, em especial para meninas (Piola et al., 2019;).

A escola pode contribuir na promoção de atividade física, espera-se que ela sirva de elemento propulsor destas iniciativas, visando facilitar a prática além das experiências que as aulas de educação física proporcionam (Camargo et al., 2017). Incorporar estratégias que aumentem a autoeficácia (por exemplo: experiências de domínio da atividade física) e reduzam sentimentos de vergonha podem colaborar na redução de barreiras (Garn et al., 2020) Não mencionado no presente estudo, mas relatado atualmente na literatura problemas de relacionamento entre adolescentes podem levar a situações de bulling e a princípio criar barreiras (Méndez et al., 2019). A esse respeito, a prática da atividade física pode promover a responsabilidade e melhora da convivência (Méndez et al., 2019). 


\section{Camargo; López-Gil \& Campos}

Meninos não foram tão consistentes com as respostas, além de: não ter companhia e preguiça, também citaram: clima, falta de locais e não ter alguém para levar. Sobre o clima, ela é uma barreira específica de cidades que possuem condições climáticas voltadas para o frio e chuva. Outros estudos realizados na cidade de Curitiba, Sul do Brasil, reportaram o clima como barreira para atividades físicas (Santos et al., 2009; Santos et al., 2010).

Ademais, um estudo de revisão sistemática sobre barreiras para a prática de atividade física na população brasileira, mencionou a falta de locais e não ter alguém para levar (Rech et al., 2018). A promoção de atividades e instalações que aumentem a interação social parece ser uma alternativa importante para aumentar os níveis de atividade física entre os adolescentes (Rech et al., 2018). O incentivo ao uso de parques e praças para promoção da atividade física, também pode contribuir para a compreensão de possíveis atividades gratuitas na comunidade (Silva et al., 2019).

Por fim, quando comparado os grupos (inativo, insuficientemente ativo, ativo), meninas insuficientemente ativas relataram significativamente com mais frequência as barreiras: acha chato, clima e não ter como pagar. Para seus pares, apenas a barreira: não ter motivação apresentou diferença significativa para o grupo de meninos inativos. É necessário, que a escola repense nas aulas de educação física como uma ferramenta de aprendizagem para o comportamento ativo e ainda, oportunize o acesso a outras praticas, para que meninas e meninos tenham acesso a atividades diversas (Camargo et al., 2017). Com o intuito de que a atividade física não seja monótona, que adolescentes sintam-se motivados a realizar, em diversos locais (casa, parque, praças) e em diversas companhias (familiares, amigos, colegas da igreja, do trabalho) o que aprenderam na escola (Camargo et al., 2017). Em especial, que os adolescentes consigam perceber as diferentes formas de realizar atividade física sem custos, como por exemplo: a utilização de espaços públicos de lazer(Silva et al., 2019). Uma vez que outros estudos também mencionaram que, não ter como pagar pode ser uma barreira para diversas populações (Vasquez et al., 2020).

Um aspecto positivo deste estudo está no uso do questionário desenvolvido por Santos et al. 2009 (Santos et al., 2009), que foi validado em adolescentes da mesma localização geográfica. Além disso, deve-se notar que, sendo uma amostra representativa e suficientemente ampla, a extrapolação dos resultados da amostra analisada adquire maior confiança. Entretanto, apesar dos pontos positivos, algumas limitações desta pesquisa devem ser levadas em consideração. Em primeiro lugar, o desenho transversal não permite estabelecer uma relação causal entre barreiras e inatividade física no lazer. Da mesma forma, o estudo foi realizado em escolas públicas, não incluindo adolescentes de escolas privadas que podem ter características diferentes da população analisada.

Pesquisas futuras poderão analisar o custoefetividade das intervenções sociais e ambientais na escola com a percepção de barreiras. Além de estudos com grupos focais direcionados para estruturações da escola, que tenham como objetivos: ampliar o nível de atividade física de adolescentes, repassar conhecimento sobre a importância de ser ativo fisicamente para os adolescentes e seus respectivos pais, elaborar estratégias junto a comunidade. Assim, novos estudos podem aumentar a compreensão sobre como reduzir a percepção de barreiras para atividade física entre adolescentes e com isso, maximizar o nível de atividade física dessa população.

\section{CONCLUSÃO}

Meninas percebem um número de barreiras superior quando comparado com meninos. "Não ter companhia" e "preguiça" foram as barreiras mais citadas por meninas em todos os grupos. Meninas insuficientemente ativas apresentaram diferenças significativas, ao reportar com mais frequência as barreiras: "acha chato", "clima" e "não tem como pagar", quando comparado com os demais grupos (inativas e ativas). Meninos reportaram com mais frequências as barreiras: "não ter alguém para levar", "preguiça”, "não ter companhia", "clima", "falta de 


\section{Barreiras para prática de atividade física}

locais". Quando comparado com os demais grupos (insuficientemente ativo e ativo) apenas a barreira "não ter motivação" apresentou diferença significativa para o grupo de meninos inativos fisicamente.

\section{APLICAÇÕES PRÁTICAS}

Profissionais do esporte e áreas afins, podem utilizar as contribuições deste manuscrito em seu trabalho profissional. Profissionais e escola podem elaborar estratégias junto a pais e toda a comunidade, visando compreender quais são as barreiras para a prática de atividade física dos adolescentes da sua escola. Visando minimiza-las. A consequência dessa ação será maiores níveis de atividade física, seguido de inúmeros benefícios para a saúde.

\section{REFERENCIAS}

1. Associação Brasileira de Empresas de Pesquisa (2016). Critério de Classificação Econômica. São Paulo, Brasil: ABEP. iṣ̌p]

2. Ato, M., López, J. J., \& Benavente, A. (2013). Un sistema de clasificación de los diseños de investigación en psicología. Anales de Psicología, 29(3), 1038-59. https://doi.org/10.6018/analesps.29.3.178511

3. Camargo, E. M., Paiva, H. K., Pacheco, H. L. M., \& Campos W. (2017). Facilitadores para a prática de atividade física no lazer em adolescentes. Revista Brasileira de Atividade Física e Saúde, 22(6), 561-67. https://doi.org/10.12820/rbafs.v.22n6p561-67

4. Dias, D. F., Loch, M. R., \& Ronque, E. R. V. (2015). Barreiras percebidas à prática de atividades físicas no lazer e fatores associados em adolescentes. Ciência e Saúde Coletiva, 20(11), 3339-50. https://doi.org/10.1590/1413$\underline{812320152011.00592014}$

5. Garn, A. C., \& Simonton, K. L. (2020). Young Adults' Short-Term Trajectories of Moderate Physical Activity: Relations With SelfEvaluation Processes. Frontiers in psychology, 11, 2079. https://doi.org/10.3389/fpsyg.2020.02079

6. Guedes, D. P., Lopes C. C., \& Guedes, J. E. R. P. (2005). Reprodutibilidade e validade do Questionário Internacional de Atividade Física em adolescentes. Revista Brasileira de Medicina do Esporte, 11(2), 151-8. http://dx.doi.org/10.1590/S1517$\underline{86922005000200011}$

7. Guthold, R., Stevens, G. A., Riley, L. M., \& Bull, F. C. (2020). Global trends in insufficient physical activity among adolescents: a pooled analysis of 298 population-based surveys with 1.6 million participants. The Lancet. Child \& adolescent health, 4(1), 23-35. https://doi.org/10.1016/S2352-4642(19)30323$\underline{2}$

8. Méndez, I., Ruiz-Esteban, C., \& Ortega, E. (2019). Impact of the Physical Activity on Bullying. Frontiers in psychology, 10, 1520. https://doi.org/10.3389/fpsyg.2019.01520

9. NCD Risk Factor Collaboration. (2017). Worldwide trends in body-mass index, underweight, overweight, and obesity from 1975 to 2016: a pooled analysis of 2416 population-based measurement studies in 128.9 million children, adolescents, and adults. Lancet, 390(10113), 2627-42. https://doi.org/10.1016/S0140-6736(17)32129$\underline{3}$

10. Organização Mundial da Saúde. (2006). Curvas de Referência da Organização Mundial da Saúde. Recuperado de: http://ebook.ecogobesity.eu/wp-

content/uploads/2017/05/ECOG-ObesityeBook-Curvas-de-referencia-da-organizacaomundial-da-saude.pdf

11. Organização Mundial da Saúde. (2018). Mais pessoas ativas para um mundo mais saudável. Plano de ação global para a atividade física 2018-2030. Recuperado de: https://apps.who.int/iris/bitstream/handle/1066 5/272721/WHO-NMH-PND-18.5-por.pdf 


\section{Camargo; López-Gil \& Campos}

12. Piola, T. S., Bacil, E., Watanabe, P., Camargo, E., Fermino, R., \& Campos, W. (2019). Sexual Maturation, social support and physical activity in adolescents. Cuadernos De Psicología Del Deporte, 19(3), 125-38. https://doi.org/10.6018/cpd.347821

13. Portela-Pino, I., López-Castedo, A., MartínezPatiño, M. J., Valverde-Esteve, T., \& Domínguez-Alonso, J. (2019). Gender Differences in Motivation and Barriers for The Practice of Physical Exercise in Adolescence. International journal of environmental research and public health, 17(1), 168. https://doi.org/10.3390/ijerph17010168

14. Prochnow, T., van Woudenberg, T. J., \& Patterson, M. S. (2020). Network Effects on Adolescents' Perceived Barriers to Physical Activity. Journal of physical activity \& health, 1-6. Advance online publication. https://doi.org/10.1123/jpah.2019-0655

15. Rech, C. R., Camargo, E. M. de, Araujo, P. A. B, Loch, M. R., \& Reis, R. S. (2018). Perceived barriers to leisure-time physical activity in the brazilian population. Revista Brasileira de Medicina do Esporte, 24(4), 303-9. http://dx.doi.org/10.1590/1517$\underline{869220182404175052}$

16. Rosselli, M., Ermini, E., Tosi, B., Boddi, M., Stefani, L., Toncelli, L., \& Modesti, P. A. (2020). Gender differences in barriers to physical activity among adolescents. Nutrition, metabolism, and cardiovascular diseases : NMCD, 30(9), $1582-1589$. https://doi.org/10.1016/j.numecd.2020.05.005

17. Santos, M. S., Hino, A. A. F., Reis, R. S., \& Rodriguez-Añez, C. R. (2010). Prevalência de barreiras para a prática de atividade física em adolescentes. Revista Brasileira de Epidemiologia, 13(1), 94-104. http://dx.doi.org/10.1590/S1415790X2010000100009

18. Santos, M. S., Reis, R. S., Rodriguez-Añez, C. R., \& Fermino, R, C. (2009). Desenvolvimento de um instrumento para avaliar barreiras para a prática de atividade física em adolescentes. Revista Brasileira de Atividade Física e Saúde, 14(2), 76-85. https://doi.org/10.12820/rbafs.v.14n2p76-85

19. Silva, A. A. P. de, Camargo, E. M. de, Silva, A. T. da, Silva, J. S. B., Hino, A. A. F., \& Reis, R. S. (2019). Caracterização das atividades físicas realizadas por adolescentes de Curitiba, Brasil. Revista Brasileira de Medicina do Esporte, 25(3), 211-5. https://dx.doi.org/10.1590/1517$\underline{869220192503188171}$

20. Tremblay, M. S., Carson, V., Chaput, J. P., Connor-Gorber, S., Dinh, T., Duggan, M., ... \& Zehr, L. (2016). Canadian 24-hour Movemnt Guidelines for children and Youth: an integration of physical activity, sedentary behaviour, and sleep. Applied Physiology Nutrition Metabolism, 41(6), S311-27. https://dx.doi.org/10.1139 / apnm-2016-0151

21. Vasquez, T., Fernandez, A., Haya-Fisher, J., Kim, S., \& Beck, A. L. (2020). A Qualitative Exploration of Barriers and Facilitators to Physical Activity Among Low-Income Latino Adolescents. Hispanic health care international : the official journal of the National Association of Hispanic Nurses, 1540415320956933. Advance online publication.https://doi.org/10.1177/154041532 0956933 


\section{Barreiras para prática de atividade física}

Tabela 2. Características dos grupos inativos fisicamente, insuficientemente ativos, ativos fisicamente (Curitiba, Paraná, Brasil - n=1518)

\begin{tabular}{|c|c|c|c|c|c|c|c|c|}
\hline \multirow[t]{2}{*}{ Variáveis } & \multicolumn{2}{|c|}{$\begin{array}{c}\text { Inativos } \\
\text { Fisicamente } \\
\leq 10 \mathrm{~min} / \mathrm{sem}\end{array}$} & \multicolumn{2}{|c|}{$\begin{array}{c}\text { Insuficientemente ativos } \\
>10 \text { até } 419 \mathrm{~min} / \mathrm{sem}\end{array}$} & \multicolumn{2}{|c|}{$\begin{array}{c}\text { Ativos } \\
\text { Fisicamente } \\
\geq 420 \mathrm{~min} / \mathrm{sem}\end{array}$} & \multicolumn{2}{|c|}{ Total } \\
\hline & $\mathrm{n}$ & $\%$ & $\mathrm{n}$ & $\%$ & $\mathrm{n}$ & $\%$ & $\mathrm{n}$ & $\%$ \\
\hline Sexo & 464 & $30.6 \%$ & 550 & $36.2 \%$ & 504 & 33.2 & 1518 & $100.0 \%$ \\
\hline Masculino & 179 & $11.8 \%$ & 221 & $14.6 \%$ & 219 & $14.4 \%$ & 619 & $40.8 \%$ \\
\hline Feminino & 285 & $18.8 \%$ & 329 & $21.7 \%$ & 285 & $18.8 \%$ & 899 & $59.2 \%$ \\
\hline Idade & 464 & $30.6 \%$ & 550 & $36.2 \%$ & 504 & 33.2 & 1518 & $100.0 \%$ \\
\hline 15 anos & 180 & $11.9 \%$ & 120 & $7.9 \%$ & 164 & $10.8 \%$ & 464 & $30.6 \%$ \\
\hline 16 anos & 97 & $6.4 \%$ & 247 & $16.3 \%$ & 157 & $10.3 \%$ & 501 & $33.0 \%$ \\
\hline 17 anos & 150 & $9.9 \%$ & 137 & $9.0 \%$ & 138 & $9.1 \%$ & 425 & $28.0 \%$ \\
\hline 18 anos & 37 & $2.4 \%$ & 46 & $3.0 \%$ & 45 & $3.0 \%$ & 128 & $8.4 \%$ \\
\hline Estado Nutricional & 464 & $30.6 \%$ & 550 & $36.2 \%$ & 504 & 33.2 & 1518 & $100.0 \%$ \\
\hline Peso Normal & 386 & $25,4 \%$ & 466 & $30.7 \%$ & 408 & $26.9 \%$ & 1260 & $83.0 \%$ \\
\hline Excesso de Peso & 78 & $5.1 \%$ & 84 & $5.5 \%$ & 96 & $6.3 \%$ & 258 & $17.0 \%$ \\
\hline Escolaridade do pai & 464 & $30.6 \%$ & 550 & $36.2 \%$ & 504 & $33.2 \%$ & 1518 & $100.0 \%$ \\
\hline Até Fundamental Completo & 165 & $10.9 \%$ & 178 & $11.7 \%$ & 167 & $11.0 \%$ & 510 & $33.6 \%$ \\
\hline Ensino Médio Completo & 182 & $12.0 \%$ & 237 & $15.6 \%$ & 209 & $13.8 \%$ & 628 & $41.4 \%$ \\
\hline Ensino Superior Completo & 117 & $7.7 \%$ & 135 & $8.9 \%$ & 128 & $8.4 \%$ & 380 & $25.0 \%$ \\
\hline Escolaridade mãe & 464 & $30.6 \%$ & 550 & $36.2 \%$ & 504 & $33.2 \%$ & 1518 & $100.0 \%$ \\
\hline Até Fundamental Completo & 171 & $11.3 \%$ & 189 & $12.5 \%$ & 157 & $10.3 \%$ & 517 & $34.1 \%$ \\
\hline Ensino Médio Completo & 172 & $11.3 \%$ & 238 & $15.7 \%$ & 220 & $14.5 \%$ & 630 & $41.5 \%$ \\
\hline Ensino Superior Completo & 121 & $8.0 \%$ & 123 & $8.1 \%$ & 127 & $8.4 \%$ & 371 & $24.4 \%$ \\
\hline$N S E$ & 464 & $30.6 \%$ & 550 & $36.2 \%$ & 504 & $33.2 \%$ & 1518 & $100.0 \%$ \\
\hline Baixo NSE & 85 & $5.6 \%$ & 110 & $7.2 \%$ & 94 & $6.2 \%$ & 289 & $19.0 \%$ \\
\hline Médio NSE & 300 & $19.8 \%$ & 331 & $21.8 \%$ & 316 & $20.8 \%$ & 947 & $62.4 \%$ \\
\hline Alto NSE & 79 & $7.2 \%$ & 109 & $7.2 \%$ & 94 & $6.2 \%$ & 282 & $18.6 \%$ \\
\hline
\end{tabular}

NSE. IIÍvet socioeconôninico. 


\section{Camargo; López-Gil \& Campos}

Tabela 3. Barreiras percebidas para a prática de atividade física no lazer segundo os grupos (inativos, insuficientemente ativos, ativos) de meninas e meninos (Curitiba, Paraná, Brasil - n=1518).

\begin{tabular}{|c|c|c|c|c|c|c|c|c|c|}
\hline \multirow[t]{2}{*}{$\begin{array}{c}\text { Barreiras } \\
\text { para meninas }(n=899)\end{array}$} & \multicolumn{2}{|c|}{$\begin{array}{l}\text { Inativos Fisicamente } \\
\leq 10 \mathrm{~min} / \mathrm{sem}\end{array}$} & \multicolumn{2}{|c|}{$\begin{array}{l}\text { Insuficientemente Ativos } \\
>10 \text { até } 419 \mathrm{~min} / \mathrm{sem}\end{array}$} & \multicolumn{2}{|c|}{$\begin{array}{c}\text { Ativos } \\
\text { Fisicamente } \\
\geq 420 \mathrm{~min} / \mathrm{sem}\end{array}$} & \multirow[b]{2}{*}{$p$} & \multicolumn{2}{|c|}{ Total } \\
\hline & $\mathrm{n}$ & $\%$ & $\mathrm{n}$ & $\%$ & $\mathrm{n}$ & $\%$ & & $\mathrm{n}$ & $\%$ \\
\hline 1 Falta de locais & 99 & $31.1 \%$ & 123 & $38.7 \%$ & 96 & $30.2 \%$ & .610 & 318 & $100.0 \%$ \\
\hline 2 Não tem alguém para levar & 93 & $31.5 \%$ & 104 & $35.5 \%$ & 98 & $33.2 \%$ & .764 & 295 & $100.0 \%$ \\
\hline 3 Não consegue realizar os movimentos & 41 & $34.5 \%$ & 39 & $32.8 \%$ & 39 & $32.8 \%$ & .630 & 119 & $100.0 \%$ \\
\hline 4 Prefere fazer outras coisas & 129 & $30.9 \%$ & 155 & $37.1 \%$ & 134 & $32.1 \%$ & .880 & 418 & $100.0 \%$ \\
\hline 5 Não tem motivação & 87 & $31.6 \%$ & 97 & $35.3 \%$ & 91 & $33.1 \%$ & .806 & 275 & $100.0 \%$ \\
\hline 6 Tem preguiça & 141 & $30.0 \%$ & 182 & $38.7 \%$ & 147 & $31.3 \%$ & .338 & 470 & $100.0 \%$ \\
\hline 7 Não tem companhia & 204 & $33.2 \%$ & 214 & $34.9 \%$ & 196 & $31.9 \%$ & .130 & 614 & $100.0 \%$ \\
\hline 8 Acha chato & 50 & $21,4 \%$ & $109^{\mathrm{a}}$ & $46,6 \%$ & 75 & $32,1 \%$ & $.013 *$ & 234 & $100.0 \%$ \\
\hline 9 Ninguém convida & 91 & $35.5 \%$ & 84 & $32.8 \%$ & 81 & $31.6 \%$ & .216 & 256 & $100.0 \%$ \\
\hline 10 Não tem tempo & 99 & $33.7 \%$ & 102 & $34.7 \%$ & 93 & $31.6 \%$ & .617 & 294 & $100.0 \%$ \\
\hline $11 \mathrm{O}$ clima dificulta & 138 & $31.6 \%$ & $174^{\mathrm{a}}$ & $39.8 \%$ & 125 & $28.6 \%$ & $.003 *$ & 437 & $100.0 \%$ \\
\hline 12 Não tem como pagar & 58 & $28.3 \%$ & $84^{\mathrm{a}}$ & $41.0 \%$ & 63 & $30.7 \%$ & $.005 *$ & 205 & $100.0 \%$ \\
\hline
\end{tabular}




\section{Barreiras para prática de atividade física}

Tabela 3. Barreiras percebidas para a prática de atividade física no lazer segundo os grupos (inativos, insuficientemente ativos, ativos) de meninas e meninos (Curitiba, Paraná, Brasil - n=1518). (cont.)

\begin{tabular}{|c|c|c|c|c|c|c|c|c|c|}
\hline \multirow[t]{2}{*}{$\begin{array}{c}\text { Barreiras } \\
\text { para meninos }(n=619)\end{array}$} & \multicolumn{2}{|c|}{$\begin{array}{l}\text { Inativos Fisicamente } \\
\leq 10 \mathrm{~min} / \mathrm{sem}\end{array}$} & \multicolumn{2}{|c|}{$\begin{array}{l}\text { Insuficientemente Ativos } \\
>10 \text { até } 419 \mathrm{~min} / \mathrm{sem}\end{array}$} & \multicolumn{2}{|c|}{$\begin{array}{c}\text { Ativos } \\
\text { Fisicamente } \\
\geq 420 \mathrm{~min} / \mathrm{sem}\end{array}$} & \multirow[b]{2}{*}{$p$} & \multicolumn{2}{|c|}{ Total } \\
\hline & $\mathrm{n}$ & $\%$ & $\mathrm{n}$ & $\%$ & $\mathrm{n}$ & $\%$ & & $\mathrm{n}$ & $\%$ \\
\hline 1 Falta de locais & 49 & $27.1 \%$ & 62 & $34.3 \%$ & 70 & $38.7 \%$ & .539 & 181 & $100.0 \%$ \\
\hline 2 Não tem alguém para levar & 57 & $30.8 \%$ & 61 & $33.0 \%$ & 67 & $36.2 \%$ & .629 & 185 & $100.0 \%$ \\
\hline 3 Não consegue realizar os movimentos & 15 & $29.4 \%$ & 17 & $33.3 \%$ & 19 & $37.7 \%$ & .929 & 51 & $100.0 \%$ \\
\hline 4 Prefere fazer outras coisas & 49 & $30.6 \%$ & 64 & $40.0 \%$ & 47 & $29.4 \%$ & .171 & 160 & $100.0 \%$ \\
\hline 5 Não tem motivação & $46^{\mathrm{a}}$ & $41.4 \%$ & 34 & $30.6 \%$ & 31 & $27.9 \%$ & $.005 *$ & 111 & $100.0 \%$ \\
\hline 6 Tem preguiça & 56 & $30.1 \%$ & 60 & $36.8 \%$ & 47 & $28.8 \%$ & .082 & 163 & $100.0 \%$ \\
\hline 7 Não tem companhia & 56 & $30.1 \%$ & 70 & $37.6 \%$ & 60 & $32.3 \%$ & .566 & 186 & $100.0 \%$ \\
\hline 8 Acha chato & 19 & $20,9 \%$ & 38 & $41,8 \%$ & 34 & $37,4 \%$ & .554 & 91 & $100.0 \%$ \\
\hline 9 Ninguém convida & 45 & $32.1 \%$ & 44 & $31.4 \%$ & 51 & $36.4 \%$ & .443 & 140 & $100.0 \%$ \\
\hline 10 Não tem tempo & 39 & $26.9 \%$ & 50 & $34.5 \%$ & 56 & $38.6 \%$ & .635 & 145 & $100.0 \%$ \\
\hline $11 \mathrm{O}$ clima dificulta & 51 & $29.0 \%$ & 68 & $38.6 \%$ & 57 & $32.4 \%$ & .545 & 176 & $100.0 \%$ \\
\hline 12 Não tem como pagar & 31 & $39.7 \%$ & 22 & $28.2 \%$ & 25 & $32.1 \%$ & .071 & 78 & $100.0 \%$ \\
\hline
\end{tabular}

${ }^{*} p<.050$ (Teste H de Kruskall-Wallis); ${ }^{a} p<.050$ (Post-hoc de Kruskall-Wallis). 